\title{
Subdiffraction Photon Guidance by Quantum-Dot Cascades
}

\author{
Chia-Jean Wang, ${ }^{\dagger}$ Ludan Huang, ${ }^{\ddagger}$ Babak A. Parviz, ${ }^{\dagger}$ and Lih Y. Lin ${ }^{\star}, \dagger$ \\ Department of Electrical Engineering, Department of Physics, \\ University of Washington, Seattle, Washington 98195
}

Received August 21, 2006; Revised Manuscript Received September 26, 2006

\begin{abstract}
We report on a waveguide composed of a cascade of gain-enabled quantum dots with subwavelength dimensions. Fabrication is demonstrated through DNA-mediated self-assembly and a two-layer molecular self-assembly process that enables rapid prototyping. The device, which is identified with fluorescence microscopy and tested by optical near field detection, allows optical signal transfer at a well-defined wavelength in flexible routing geometry, such as straight paths and $90^{\circ}$ bends. The structure serves as a critical building block for nanophotonic systems with high integration density.
\end{abstract}

Realization of ultrahigh-density photonic integrated circuits requires the ability to guide light at subdiffraction limit dimensions without extensive loss due to sharp waveguide bending. To fully capture the inherent advantages of large bandwidth, capacity, and high-speed modulation in optical communication at the submicrometer scale, structures such as the silicon-on-insulator high index contrast, ${ }^{1}$ onedimensional negative dielectric, ${ }^{2}$ photonic crystal, ${ }^{3,4}$ nanowire, and nanoribbon ${ }^{5}$ waveguides provide a wealth of options. However, the same techniques may still be subjected to the diffraction limit, may be difficult to integrate with optical and electronic components on chip, and cannot maintain low-loss guiding at sharp junctions. Alternatively, metal nanoparticle arrays, which are made of $\mathrm{Ag}, \mathrm{Au}$, or $\mathrm{Al}$ assembled through sputtering, ion implantation, thermal evaporation, and lift-off, ${ }^{6-9}$ present a nanoscale method of guiding energy via plasmon excitation. Still, optical energy transmission through these metal nanoparticles is subject to resistive heating and loss and necessitates conversion to translate plasmon energy back to optical energy. In keeping with the low dimensionality of nanoparticles and harnessing a gain-enabled material, we elaborate upon the principle of operation, methods for fabrication, and demonstration of a quantum-dot waveguide including both straight and corner formations. The device expands the toolbox of nanophotonic components to further allow energy transmission over sharp bends and through confined spaces.

The quantum dot (QD), which exhibits three-dimensional (3D) electron-hole $\left(\mathrm{e}^{-}-\mathrm{h}^{+}\right)$pair confinement leading to sharp emission peaks largely tuned by particle size and composition, have enabled applications spanning detection

\footnotetext{
* Corresponding author. E-mail: lin@ee.washington.edu.

$\dagger$ Department of Electrical Engineering.

$\doteqdot$ Department of Physics.
}

of biomolecules ${ }^{10,11}$ to lasers with lower threshold current density compared to those made from bulk material. ${ }^{12-14}$ In colloidal form, QDs may be modified with various surface chemistries useful for self-assembly processes requiring precise attachment schemes. Furthermore, in an appropriate semiconductor system, the particle reveals a gain mechanism that uses a pump source to create population inversion of excited electrons to generate net emission over absorption, similar to optical amplifying action. ${ }^{15}$ Consequently, the propagating optical signal may not only experience less transmission loss but increase in strength as well. Capitalizing on the specified advantages, the quantum-dot waveguide consists of an array of densely packed quantum dots anchored to a substrate via self-assembly. Operation of the device, depicted in Figure 1A, requires a pump laser of photon energy greater or equal to the separation of the second electron and hole states to be placed overhead to excite electron-hole pairs and enable gain. For a three-state system, the $\mathrm{e}^{-}-\mathrm{h}^{+}$pairs quickly relax to the first conduction- and valence-band states, where without further manipulation they will likely recombine for spontaneous emission of photons. ${ }^{16,17}$ However, aligning a signal laser of energy equivalent to separation between the first electron and hole state to the input edge of the waveguide, photons generated by stimulated emission may cascade through the device creating an amplified output, moderated by the interdot coupling efficiency and the gain available in each dot. The FDTD simulation of the Poynting vector distribution, indicating photon transmission behavior in a QD cascade waveguide, is provided in Figure 1B. ${ }^{18}$ The parameters match test conditions such that we use 10-nm-diameter quantum dots, which approximate the size of the core/shell nanoparticles, and an input signal with wavelength at $633 \mathrm{~nm}$ and polarization in the $y$ direction. Upon absorption of both pump 
A
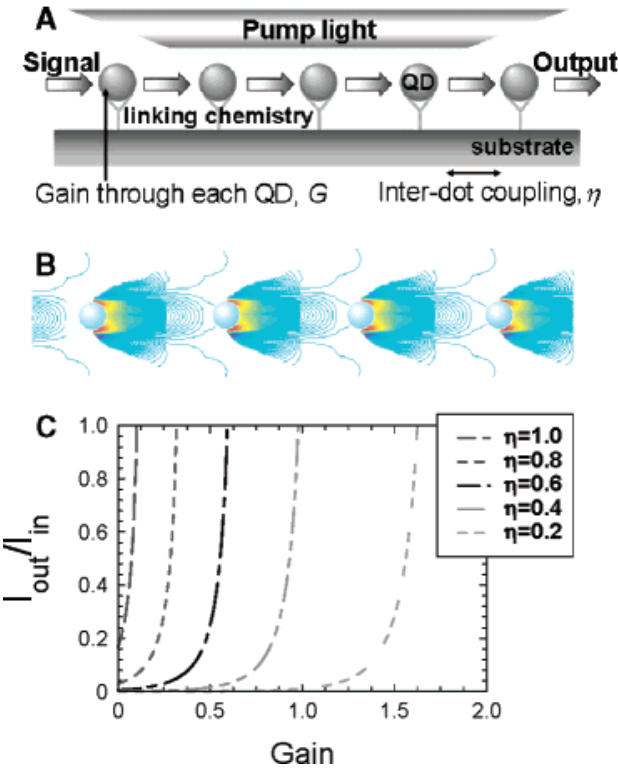

Figure 1. QD nanophotonic waveguide. (A) Operation: a pump source excites electrons to the second energy state in the conduction band of the quantum dot to generate gain, and a signal light corresponding to the first excited state is placed at the waveguide edge to cause stimulated emission of photons that propagate downstream to produce an output signal mediated by the inter-dot coupling. (B) Poynting vector distribution of the quantum-dot cascade. (C) Calculated relative output intensity as a function of gain per quantum dot for a 1D array waveguide using varied coupling coefficients, $\eta$.

and signal light, the accepting QDs produce energy as photons via stimulated emission, with the majority of energy aimed in the propagation direction to be absorbed by the neighboring QD causing a cascade of energy transmission. Hence, the Poynting vector magnitude illustrates that the power per unit area lies in the path of forward propagation.

Compared to other possible analogous designs where QDs are formed through lattice mismatch between epitaxially grown layers ${ }^{19}$ and waveguides are defined by etching the dielectric layer, the proposed design achieves simpler fabrication and eliminates the minimum width constraint to achieve guided mode approximated by the transmission wavelength from conventional dielectric waveguiding. To note, prior modeling of the gain response for $5 \times 5 \times 5$ $\mathrm{nm}^{3} \mathrm{CdSe} / \mathrm{ZnS}$ core/shell quantum dots, with an additional $5 \mathrm{~nm}$ shell thickness, indicates that there is net gain occurring between 0.1 and $1 \mathrm{nW}$ optical pump power, with absorption dominating at the low end and gain saturation at the higher end. ${ }^{17}$ In addition, implementing an $\mathrm{ABCD}$ transfer matrix technique allows estimation of the relative output intensity with respect to the photon coupling coefficient between two adjacent QDs and the gain parameter inherent in each. As an example, the simulation results for a waveguide composed of five quantum dots in a 1D array (Figure 1C) indicate a tradeoff between the gain and coupling coefficient where a smaller value for one may compensate a higher value for the other to attain high transmission efficiency.

Fabrication of our nanophotonic waveguides has been accomplished through both DNA-mediated self-assembly and a simpler two layer molecular self-assembly procedure
A
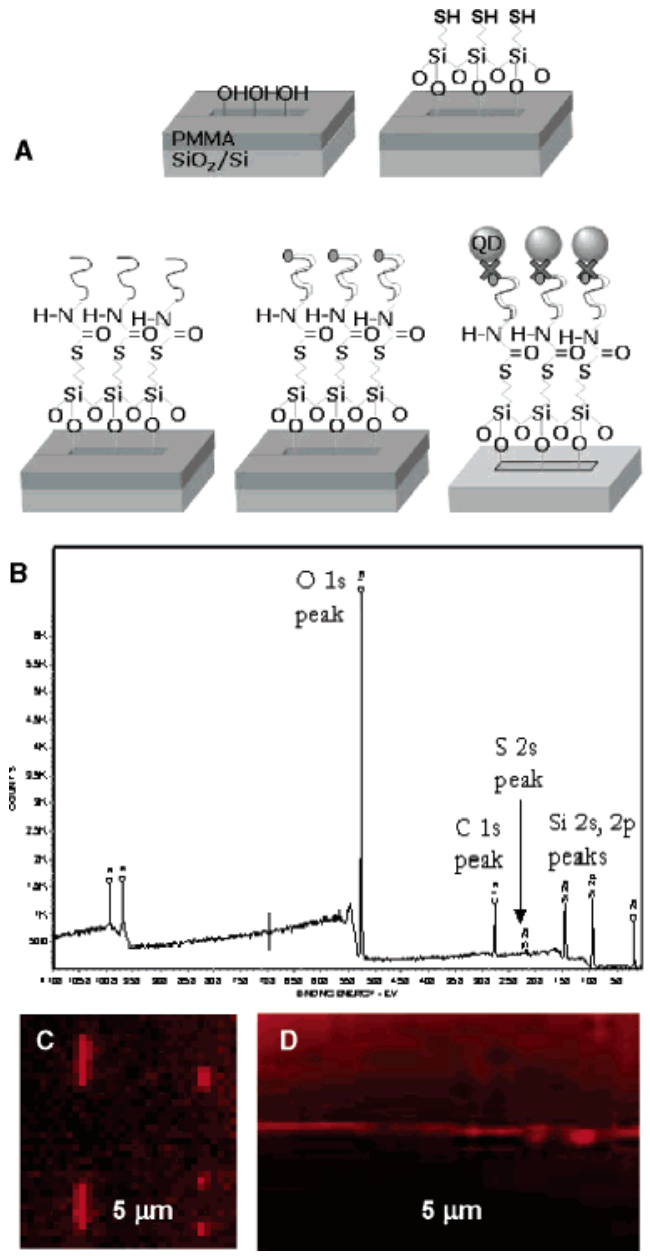

Figure 2. DNA-mediated self-assembly of QD waveguides. (A) EBL pattern PMMA-coated substrate, treat with $\mathrm{O}_{2}$ plasma; deposit MPTMS monolayer; covalently bind with 5'-acrydite-DNA; hybridize with biotin-modified cDNA; bind streptavidin-QDs to biotincDNA sites, remove PMMA with dichloromethane. (B) XPS results confirming MPTMS deposition through sulfur content on substrate. (C) Fluorescence micrograph of $5 \mu \mathrm{m} \times 1 \mu \mathrm{m}, 2 \mu \mathrm{m} \times 1 \mu \mathrm{m}$, and $1 \times 1 \mu \mathrm{m}^{2}$ patterns and (D) $1-\mu \mathrm{m}$-width waveguide.

relying on specific covalent bonds. The DNA process enables programmable deposition of quantum dots to serve as a template for simultaneous assembly of multiple QD type waveguides. The procedure, shown in Figure 2A, begins with spin-coating polymethylmethacrylate (PMMA) on a silicon/ silicon dioxide substrate and using electron-beam lithography (EBL) to pattern trenches on the surface of PMMA, thus defining the global shape of the waveguide. We increase the number of exposed hydroxyl groups at the bottom of these trenches by a short exposure to oxygen plasma. The $-\mathrm{OH}$ groups act as an anchor for gas-phase self-assembly of 3-mercaptopropyltrimethoxysilane (MPTMS) molecules that provide exposed thiol $(\mathrm{SH})$ termini for the subsequent binding step. The introduction of acrylamide-modified singlestranded DNA (ss-DNA) to the thiol groups enables specific binding of the ss-DNA to the bottom of the PMMA trenches, which is then free to bind to the exact complimentary chain creating a highly selective binding location. Prior to the DNA hybridization step, immersion of the sample in buffered acrylic acid passivates the nonreacted MPTMS ${ }^{20}$ and then a 
droplet of the custom-synthesized biotinylated complementary DNA (cDNA) solution is placed onto the surface. Next, we introduce $655 \mathrm{~nm}$ emission quantum dots conjugated to streptavidin, a protein that preferentially attaches to biotin. In the final step, a dichloromethane rinse dissolves the PMMA to leave the QD pattern secured to the substrate by linking chemistries. To check the deposition of the selfassembled molecular (SAM) layers, we compared exposed to control samples using X-ray photoelectron spectroscopy (XPS). Only the surfaces treated with MPTMS exhibit a sulfur signature (in Figure 2B), with the average percent compositions at $58.6 \%$ oxygen, $28.3 \%$ silicon, $12.4 \%$ carbon, and $0.7 \%$ sulfur. Alternatively, the untreated samples yielded an average of $63.0 \%$ oxygen, $29.6 \%$ silicon, and $7.4 \%$ carbon. Further assembly steps using DNA are confirmed with fluorescence observation and examples of the final QD fluorescence patterns, in Figure $2 \mathrm{C}$ and D, include an array of rectangles ranging in size from $5 \mu \mathrm{m} \times 1 \mu \mathrm{m}$ down to 1 $\times 1 \mu \mathrm{m}^{2}$, and a $1-\mu \mathrm{m}$-wide waveguide.

The second technique diverges from the DNA-based procedure after the generation of $\mathrm{Si}-\mathrm{OH}$ groups on the surface. Instead, we deposit 3'-aminopropyltriethoxylsilane (APTES) by immersion in solution to create a surface expressing amines for further chemical reactions. ${ }^{21} \mathrm{~A}$ droplet of carboxylated quantum dots suspended in 1x phosphate buffer solution (PBS) mixed with 1-ethyl-3-(3-dimethylaminopropyl)-carbodiimide (EDC), an amine-carboxyl coupling reagent, is applied to the sample as the second layer. After waiting for a minimum of $1 \mathrm{~h}$, during which the QDs bind to the sample, excess material is rinsed off with 1x PBS buffer and the PMMA is also removed with dichloromethane to reveal the waveguides. As confirmation of process, XPS in Figure 3B reveals APTES deposition such that the resulting percent compositions are $1.2 \%$ nitrogen, $29.8 \%$ silicon, $54.5 \%$ oxygen, and $14.4 \%$ carbon, whereas the control gave an average of $60.6 \%$ oxygen, $32.3 \%$ silicon, and $7.1 \%$ carbon. The ratio of the nitrogen peak to the other dominant elements is appropriate to coverage by APTES, and lack of nitrogen in controls provides further verification. Fluorescence micrographs and AFM images, in Figure 3C and $\mathrm{D}$, show attachment of $655 \mathrm{~nm}$ emission quantum dots aligned in the 100- and 500-nm-width lines. An additional atomic force micrograph is shown in Figure 3C to provide more details on the QD shape and distribution within the waveguide. To note, the $100 \mathrm{~nm}$ waveguides are diffractionlimited in terms of optically detecting discontinuities. However, the topographical profile demonstrates a continuous structure. It is noted that advanced optical lithography can be used for defining the waveguide patterns in both fabrication methods for mass production, if the resolution is sufficient. In either case, the waveguide is defined by the design file and EBL where nominal tolerance is within 5\% of the specified linewidths. Because the APTES method is subject to fewer intermediate stages, the packing is denser where QDs are separated by about 5-10 $\mathrm{nm}$ where that for DNA may range up to an order of magnitude more. More investigation is required to extract the coupling coefficients in the 2D waveguide as for our case because of the
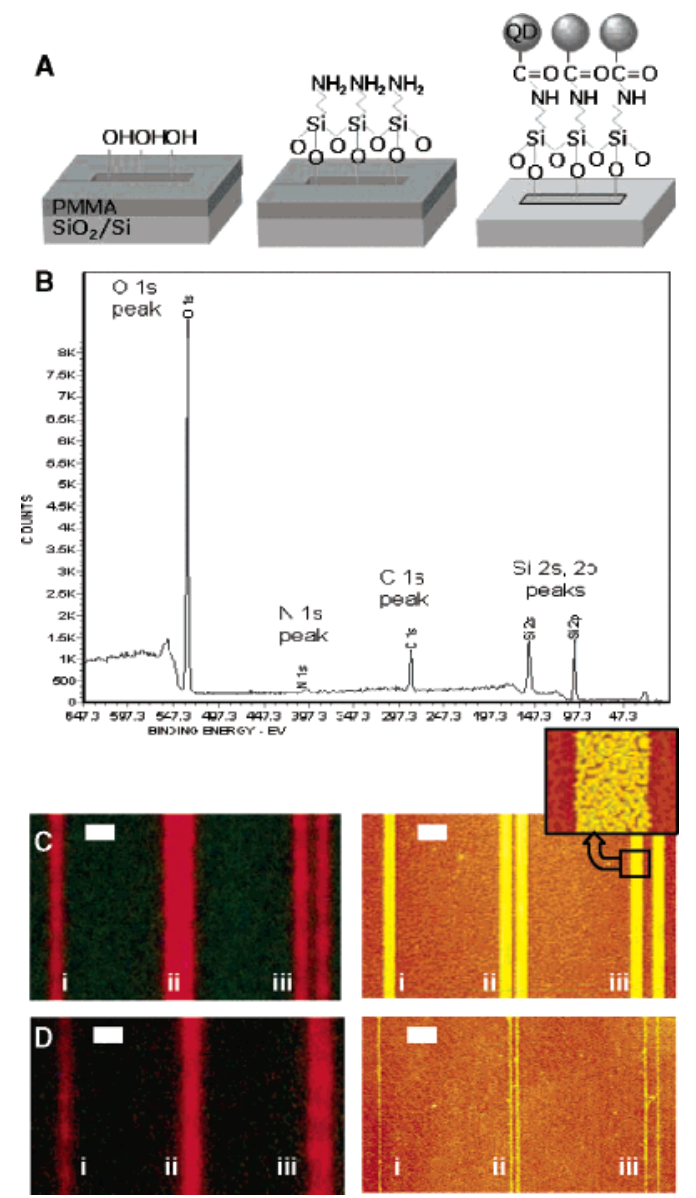

Figure 3. Two-layer self-assembly of QD waveguides. (A) EBL pattern PMMA-coated substrate, treat with $\mathrm{O}_{2}$ plasma; deposit APTES monolayer; covalently bind carboxylated QDs to the amine terminal group, remove PMMA with dichloromethane. (B) XPS confirming APTES deposition through nitrogen content. Fluorescence and corresponding atomic force micrograph of (C) $500 \mathrm{~nm}$ with inset depicting granular QD shape and (D) $100 \mathrm{~nm}$ wide waveguides in (i) single and pair formations spaced (ii) $200 \mathrm{~nm}$ and (iii) $500 \mathrm{~nm}$ apart. Scale bar is $1 \mu \mathrm{m}$ in length.

complexity caused by variation of separation between QDs at a range of directions in the forward path.

The waveguiding behavior of the quantum-dot cascades was determined by aligning a pair of tapered fiber probes to the device. Coarse adjustment was done with the aid of gold alignment markers, and fluorescence imaging of the waveguide under pump light illumination allowed us to precisely identify the device and further tune the probe position. The designated input fiber is coupled to a $639 \mathrm{~nm}$ signal laser, which falls within the gain peak centered at $655 \mathrm{~nm}$, and the output taper connects to a photodetector with a nanowatt detection limit. The pump light, with $405 \mathrm{~nm}$ wavelength, is collimated and oriented overhead to illuminate the device and excite $\mathrm{e}^{-}-\mathrm{h}^{+}$ pairs to create gain. With a 20x objective and the collimator in place, we calculated a maximum pump-power distribution of $1.4 \mathrm{nW} / \mathrm{QD}$, corresponding to $4 \mathrm{~mW}$ over a $20-\mu \mathrm{m}$ diameter illumination area, which is within the gain regime determined by the theoretical model. ${ }^{17}$ Subsequently, ramping the pump level up and down while logging the photodetector 
A pump power per QD (nW)

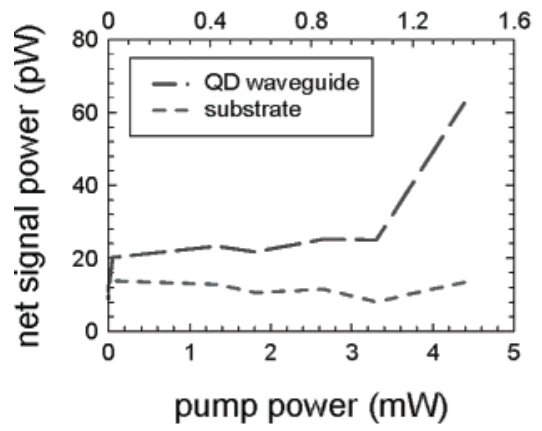

C pump power per QD (nW)

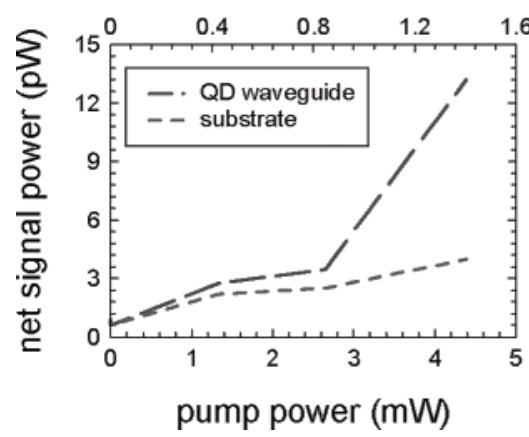

B pump power per QD (nW)

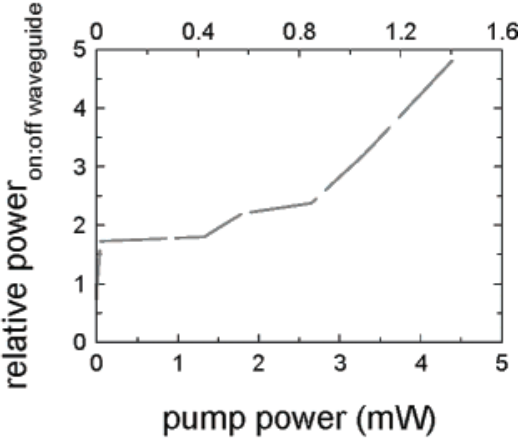

D pump power per QD (nW)

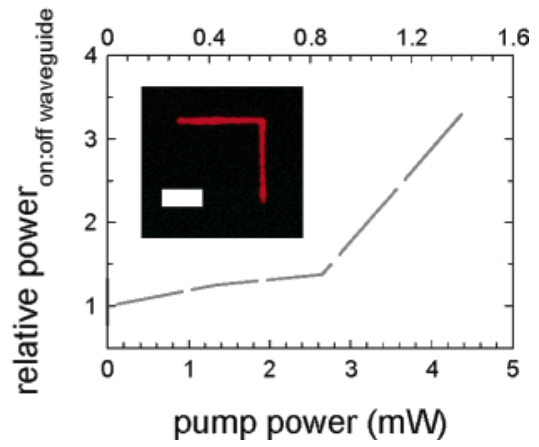

Figure 4. Measured transmission results from 500-nm-width waveguides demonstrating higher signal on the waveguide than on substrate. Straight $(10 \mu \mathrm{m})$ waveguide results: (A) net signal power and (B) resulting relative power ratio. Corner $\left(10 \times 10 \mu \mathrm{m}^{2}\right)$ waveguide: $(\mathrm{C})$ net signal power and (D) resulting relative power ratio. The inset of $\mathrm{D}$ is the fluorescence micrograph of the corner waveguide; the scale bar is $5 \mu \mathrm{m}$ in length.

measurements over time, with the signal laser on and off and the fibers aligned on and then off the waveguide, for a total of four cycles allowed us to determine the effect of the waveguide in transmitting light.

Each combination of pump and signal parameters is polled for every 5 seconds over a 10-min duration with the collection of values averaged for further analysis. On the whole, the standard deviation is 2 orders of magnitude less than the mean. The effect of fluorescence from the QDs is removed from the total level by subtracting the signal-offwaveguide from signal-on-waveguide readings at each pump power. Then, the resulting on-waveguide reading may be divided by the off-waveguide value to derive the effect of the quantum-dot cascades such that a parallel trend where increased pump power produces higher waveguide-tosubstrate ratios indicates transmission and guiding of light by the waveguide. The first $5 \mathrm{~min}$ of data are removed to account for signal laser stabilization and the outliers, which are defined as being more than one standard deviation from the mean of the raw data, are filtered from the data set. The results for straight $10-\mu \mathrm{m}$-long and $10 \mu \mathrm{m} \times 10 \mu \mathrm{m}$ corner 500 -nm-wide waveguides made by the two-layer selfassembly fabrication process are provided in Figure 4A-D, respectively. Although the width dimension at $500 \mathrm{~nm}$ is much larger than the QD diameter, signal transmission does not occur as through a regular dielectric waveguide but rather by a sequence of stimulated emission through the collection of 3D confined nanocrystals. The behavior follows the expected pattern such that the transmitted optical power is greater with the waveguide than without the waveguide given sufficient pump power. The net signal on the waveguide is consistently higher than that on the substrate and, in addition, the ratio increases with the pump power in both cases, implying a gain mechanism. Otherwise, the QD would have high absorption, and the measured output power would be lower than that without the waveguide, as in the lower pump power regions of Figure $4 \mathrm{~A}-\mathrm{D}$. It is expected that the ratio for the cornered waveguide is lower than that for the straight waveguide because of its longer length and $90^{\circ}$ bend. Compared to prior calculation, ${ }^{17}$ the QD exhibits gain starting around $0.02 \mathrm{nW}$ pump power per QD, which is indicated in the sharp rise in signal above threshold. The similar trend of increased ratio between the cornered waveguide and the straight waveguide shows the promise of extremely flexible routing using the proposed self-assembled colloidal quantumdot waveguides. The versatility of the design is one main advantage compared to conventional dielectric waveguides besides subdiffraction waveguiding, and is important for realizing extremely efficient and compact photonic integrated circuits.

In summary, we have introduced two different selfassembly fabrication methods for colloidal quantum-dot waveguides, where the DNA-mediated process provides an element of programmability and the two-layer procedure allows for rapid prototyping and optimization. Testing of the APTES-based devices shows that similar behaviors and relative waveguiding intensities exist between straight and corner patterns with subdiffraction limit dimensions where the transmitted signal levels generally increase with pump power. Both techniques may be extended toward deposition 
of multiple quantum-dot-type structures to reduce crosstalk between waveguides. ${ }^{22}$ Since gain in quantum dots is subject to pump and signal wavelengths, for two structures each tuned to transmit light at different wavelengths, overlap in signal transmission from one waveguide to another is highly suppressed. Investigation of such crosstalk contribution is an area of continuing work and will further illuminate the effectiveness of the device as a gain-enabled and wavelengthselective nanophotonic waveguide.

Acknowledgment. We gratefully acknowledge funding from the National Science Foundation (NSF) ADVANCE program, NSF award no. 0524648, and the UW Royalty Research Fund. C.-J.W. thanks the NSF Graduate Fellowship Program for financial support. Work was performed in part at the University of Washington Nanotech User Facility (NTUF), a member of the National Nanotechnology Infrastructure Network (NNIN), which is supported by the National Science Foundation.

\section{References}

(1) Barrios, C. A.; Almeida, V. R.; Panepucci, R. P.; Lipson, M. IEEE J. Lightwave Technol. 2003, 21, 2332.

(2) Takahara, J.; Yamagishi, S.; Taki, H.; Morimoto, A.; Kobayashi, T. Opt. Lett. 1997, 22, 475.

(3) Bogaerts, W.; Baets, R.; Dumon, P.; Wiaux, V.; Beckx, S.; Taillaert, D.; Luyssaert, B.; Van Campenhout, J.; Bienstman, P.; Van Thourhout, D. IEEE J. Lightwave Technol. 2005, 23, 401.

(4) Johnson, S. G.; Villeneuve, P. R.; Fan, S.; Joannopoulos, J. D. Phys. Rev. B. 2000, 62, 8212.

(5) Law, M.; Sirbuly, D. J.; Johnson, J. C.; Goldberger, J.; Saykally, R. J.; Yang, P. Science 2004, 305, 1269.
(6) Brongersma, M. L.; Hartman, J. W.; Atwater, H. A. Phys. Rev. B 2000, 62, R16356.

(7) Maier, S. A.; Barclay, P. E.; Johnson, T. J.; Friedman, M. D.; Painter, O. Appl. Phys. Lett. 2004, 4, 3990.

(8) Maier, S. A.; Kik, P. G.; Atwater, H. A.; Meltzer, S.; Harel, E.; Koel, B. E.; Requicha, A. A. G. Nat. Mater. 2003, 2, 229.

(9) Yatsui, T.; Nomura, W.; Ohtsu, M. Nano Lett. 2005, 5, 2548.

(10) Chan, W. C. W.; Nie, S. Science 1998, 281, 2016.

(11) Bruchez, M., Jr.; Moronne, M.; Gin, P.; Weiss, S.; Alivisatos, A. P. Science 1998, 281, 2013.

(12) Ledentsov, N. N.; Grundmann, M.; Heinrichsdorff, F.; Bimberg, D.; Ustinov, V. M.; Zhukov, A. E.; Maximov, M. V.; Alferov, Z. I.; Lott, J. A. IEEE J. Sel. Top. Quantum Electron. 2000, 6, 439.

(13) Ustinov, V. M.; Zhukov, A. E.; Egorov, A. Y.; Maleev, N. A. Quantum Dot Lasers; Oxford University Press: Oxford, 2003.

(14) Cao, H.; Xu, J. Y.; Xiang, W. H.; Ma, Y.; Chang, S.-H.; Ho, S. T.; Solomon, G. S. Appl. Phys. Lett. 2000, 76, 3519.

(15) Asada, M.; Miyamoto, Y.; Suematsu, Y. IEEE J. Quantum Electron. 1986, 22, 1915.

(16) Klimov, V. I.; Mikhailovsky, A. A.; Xu, S.; Malko, A.; Hollingsworth, J. A.; Leatherdale, C. A.; Eisler, H.-J.; Bawendi, M. G. Science 2000, 290, 314.

(17) Wang, C.-J.; Lin, L. Y.; Parviz, B. A. IEEE J. Sel. Top. Quantum Electron. 2005, 11, 500.

(18) Huang, L.; Wang, C.-J.; Lin, L. Y. Opt. Lett., submitted for publication.

(19) Park, G.; Shchekin, O. B.; Huffaker, D. L.; Deppe, D. G. IEEE Photonics Technol. Lett. 2000, 13, 230.

(20) Hu, W.; Sarveswaran, K.; Lieberman, M.; Bernstein, G. H. IEEE Trans. Nanotechnol. 2005, 4, 312.

(21) Demers, L. M.; Ginger, D. S.; Park, S.-J.; Li, Z.; Chung, S.-W.; Mirkin, C. A. Science 2002, 296, 1836.

(22) Wang, C.-J.; Lin, L. Y.; Parviz, B. A. Conference on Lasers and Electro-Optics (CLEO), 2006, QML7.

NL061958G 